\title{
INFLUENCE OF CLINICAL REMOUNTING OF COMPLETE DENTURES ON OCCLUSION AND DISOCCLUSION TIMES AS INDICATORS OF OCCLUSAL BALANCE: A COMPUTERISED OCCLUSAL ANALYSIS
}

\author{
Amal Ali Swelem*
}

\begin{abstract}
Background: Occlusal balance in complete dentures is usually achieved by eliminating occlusal discrepancies through selective grinding. The main challenge lies in the accurate detection of the premature contacts to be able to achieve true and proper occlusal balance. There are several ways to detect occlusal errors and premature contacts including the intra-oral clinical assessment methods and remounting procedures.
\end{abstract}

Purpose: Dental literature lacks studies that objectively investigated the accuracy of occlusal adjustment after clinical remounting procedures as compared with that done only by intra-oral assessment using articulating paper. The aim of this study was to quantitatively and objectively assess the influence of clinical remounting on occlusion (OT) and disocclusion (DT) times, as objective indicators of occlusal balance, using computerized occlusal analysis.

Methods: This clinical study included 20 completely edentulous patients, 11 males and 9 females (aged 51 to 64, with an average of 57.6 years). Two sets of conventional complete dentures were constructed for each patient with a total of 40 dentures. Occlusal adjustment of 20 denture sets was done based on intra-oral assessment using articulating paper (control group) while occlusal adjustment of the other 20 denture sets was done based on clinical remounting procedures (experimental group). Occlusal parameters including OT, DT during right excursion (DT-Right) and DT during left excursion (DT-Left) were recorded for each set of dentures. Paired t-test was used to statistically analyze and compare the OT, DT-Right and DT-Left values between the two denture groups. A probability value of less than 0.05 was considered statistically significant.

Results: The mean recorded OT was 0.48 seconds in the intra-oral method (articulating paper) group and decreased to 0.32 seconds in the clinical remounting group. This decrease was statistically significant. The mean recorded DT was 0.6 and 0.62 seconds for DT-Right and DT-Left respectively in the intra-oral method group. However they decreased to 0.36 and 0.37 seconds in the clinical remounting group. Again this decrease was statistically significant for both right and left lateral excursions.

Conclusion: Occlusal adjustment based on clinical remounting procedures is more accurate and achieves more occlusal balance, as represented by shorter occlusion and disocclusion times, when compared to the intra-oral adjustment method using an articulating paper.

*Associate Professor, Removable Prosthodontic Department, Faculty of Oral and Dental Medicine, Cairo University, Egypt. 


\section{INTRODUCTION}

One of the primary objectives of a complete denture, besides improving aesthetics and phonetics, is to restore the masticatory function of edentulous patients. This necessitates the establishment of a balanced and harmonious occlusion. ${ }^{1}$ Balanced occlusion does not only play a fundamental role in effective mastication, but it is also essential for TMJ health and the preservation of the remaining supporting structures including the residual ridge and soft tissue. ${ }^{2-4}$ Furthermore, the occlusal relationship between artificial teeth significantly affects denture stability as well as uniform loading of denture foundations..$^{5}$ Occlusal harmony was also found to decrease patient adaptation time, significantly reduce the incidence of tissue irritation and the number of post-insertion visits ${ }^{6}$, and hence increase patient's comfort ${ }^{2,6}$ and satisfaction ${ }^{7,8}$ with their dentures.

Occlusal discrepancies with new complete dentures can result from warping of the record bases, errors in mounting procedures, processing changes or errors in recording the maxillomandibular relationship. ${ }^{9}$ Occlusal balance is usually achieved by eliminating these occlusal discrepancies that represent themselves as interceptive or premature contacts ${ }^{3,8}$ through selective grinding. The main challenge here lies in the accurate detection of these interceptive contacts to be able to achieve true and proper occlusal balance and occlusal contact simultaneity. There are several ways to detect occlusal errors and premature contacts including intra-oral clinical assessment methods and remounting procedures. ${ }^{2}$

For intra-oral clinical assessment, the most commonly used method involves the use of an articulating paper. As for remounting, there are laboratory and clinical remounting procedures. Laboratory remounting aids in the detection of occlusal errors due to laboratory and processing changes, while clinical remounting detects occlusal errors caused by clinical procedures. ${ }^{3,9}$ Qualitative laboratory remounting procedures that are carried out prior to removal of dentures from their definitive casts can minimize occlusal errors however cannot eliminate them completely. ${ }^{3}$ On the other hand, a clinical remount procedure at the time of denture insertion allows correction of occlusal errors after recording maxillomandibular relationships. ${ }^{9}$ Clinical remounting seems to play a vital role in occlusal equilibration. In their recent study, Jandial et al ${ }^{10}$ found a highly significant relationship between the absence of clinical remounting and occlusal disharmony. It is believed that clinical remounting minimizes the errors due to the viscoelastic nature of the mucosa and enhances the visual detection of occlusal discrepancies which in turn allows for more accurate occlusal adjustments. ${ }^{3}$ Most dentists and laboratory technicians perform laboratory remounting. Unfortunately, only few clinicians perform clinical remounting because it is time consuming. ${ }^{2,11-13}$ Although several researchers introduced ways to simplify the procedure and make it less time-consuming ${ }^{4,12,14}$, most clinicians still use the articulating paper method instead because it is comparably quicker and simpler. 7,11,12, 14, 15

Intraoral occlusal adjustment based on articulating paper marks may be indeed faster but it has been reported to be much less accurate than occlusal correction that is based on registering a centric relation record and remounting the finished dentures. 3, 5, 12, 16, 17 Recently, Mpungose et al, ${ }^{18}$ investigated the accuracy and reliability of interpreting occlusal markings made by articulating paper on complete dentures intraorally. Clinical teachers at a training hospital subjectively interpreted occlusal markings intended for adjustment. Their scores were compared to a control score to determine accuracy. Only between $20 \%$ and $30 \%$ of observations were found to be both accurate and reliable. The authors concluded that unless the procedure can be standardized, this technique should not be considered an appropriate protocol 
for balancing occlusion of complete dentures. However, their conclusions were based on subjective assessments. To draw more definitive conclusions, accuracy of occlusal equilibration after occlusal adjustments made based on the two methods, intraoral assessment using articulating paper and clinical remounting, needs to be verified and confirmed by objective and quantitative research.

For several years now, objective assessment of occlusal equilibration has been possible through computerized occlusal analysis. The T-Scan, a quantitative occlusal analysis system, was developed by Maness et al ${ }^{19}$ in 1987. Since then the system has undergone substantial hardware and software modifications. The T-Scan III system available today has been immensely enhanced over the earliest T-Scan versions. ${ }^{20,21} \mathrm{~T}$-Scan III offers a dynamic comprehensive visual assessment of a patient's occlusion from initial tooth contact till maximum intercuspation (MI). Occlusal interferences and premature contacts in the dynamic occlusion could be accurately identified. The system is capable of measuring the timing and relative intensity of occlusal force for each tooth. Force distribution per tooth, as well as, in the two halves of the jaw can be calculated and displayed. Force distribution can also be separated into anterior and posterior segments. Moreover, the system comprises centre of force analysis where the pattern of the contacts and corresponding forces over time can be represented and the centre of force at each time-point is indicated. The system displays all this data into easily interpreted colour coded 2-D and 3-D graphics. ${ }^{20,22}$ Reliability, sensibility and reproducibility of the T-scan III system has been reported by several researchers. ${ }^{20,21,23-26}$ According to Koos et al ${ }^{20}$, the system is reliable (97.2\%) and considerably accurate (98\%) for intra-oral use and everyday clinical practice with a measurement error of $1 \%$.

The main advantage of this system however is that it allows for quantitative, hence objective, assessment of occlusal balance by recording timed occlusal contacts and displaying numerical values for occlusion (OT) and disocclusion (DT) times. OT represents the time from the first contact of occluding teeth to MI and DT is the time from MI to complete disocclusion of all posterior teeth during lateral movement. ${ }^{27-29}$ The clinical goal is to reach an OT of 0.2 seconds ${ }^{30}$ and a DT of 0.4 seconds. ${ }^{31}$ OT and DT could be considered as objective indicators of occlusal harmony and balance because a shorter OT indicates the presence of fewer pre-maturities as less time is required for all teeth to contact as the patient closes into MI, hence indicating more occlusal contact simultaneity and equilibration.

One study investigated the relationship between articulation paper mark size (captured using an intraoral photographic procedure) and the percentage of force measured with computerized occlusal analysis using the T-scan III. ${ }^{32}$ Another study compared the subjective interpretation of articulating paper markings by clinicians of different years of clinical experience with computerized occlusal analysis relative occlusal force measurements of the same tooth contacts using the T scan III system Kerstein and Radke. ${ }^{26}$ To the best of the author's knowledge however, only one study assessed occlusal equilibration of complete dentures before and after clinical remounting using computerised occlusal analysis. However this study included only 10 patients who received dentures with non-anatomic teeth arranged with a compensatory curve. There was no comparison with the conventional articulating paper method and the assessment was done using an old version of the T-scan system where the authors had to manually calculate the load distribution between the right and left sides of the jaws to assess occlusal equilibration. ${ }^{33}$

Dental literature lacks studies that objectively investigated the accuracy of occlusal adjustment after clinical remounting procedures as compared 
with that done only by intra-oral assessment using articulating paper. Hence the aim of the current study was to quantitatively and objectively assess the influence of clinical remounting on OT and DT, as objective indicators of occlusal balance, in complete denture wearers using the T-Scan III computerized occlusal analysis system. The null hypothesis of the study was that there would be no significant differences in occlusal balance achieved through occlusal adjustment based on clinical remounting as compared with that based on intraoral assessment (articulating paper method).

\section{MATERIALS AND METHODS}

\section{Patient selection}

This clinical study included 20 completely edentulous patients, 11 males and 9 females (aged 51 to 64 , with an average of 57.6 years), who were selected according to the following criteria: Had a class I maxillomandibular relationship, free from temporomandibular disorders, free from any systemic diseases that could affect neuromuscular behaviours or under any medication that could affect muscular activities. Edentulous condition of all patients fell within Class I and II cases based on the Prosthodontic Diagnostic Index (PDI) classification system for complete edentulism. ${ }^{34}$ Exclusion criteria were the presence of major medical problems or severe chronic diseases, poor residual ridge anatomy or any intra-oral soft or hard tissue pathology. All patients were given detailed information about the research and gave written informed consents for their participation.

\section{Prosthodontic procedures}

Two sets of conventional complete dentures were constructed for each patient with a total of 40 dentures. Occlusal adjustment of 20 denture sets was done based on intra-oral assessment using articulating paper (control group) while occlusal adjustment of the other 20 denture sets was done based on clinical remounting procedures (experimental group).

All laboratory work was done by the same dental technician in the same lab. All clinical steps were done based on a standardized protocol as follows: Preliminary impressions were made using irreversible hydrocolloid (Cavex CA37: Cavex Holland BV, Holland) in properly selected and modified stock trays. Final impressions were made using rubber base impression material (Coltene speedex dental vertriebs Gmnbtt Konstan Germany) in properly adjusted and border molded (Kerr, Salerno, Italy) special trays (Cold cure denture base material, Acrostone, England.). Impressions were boxed and poured in dental stone to obtain master casts on which occlusion blocks were fabricated. The maxillary occlusion rim was modified to provide adequate lip support and adjusted for proper occlusal plane orientation. A maxillary face bow (Whip Mix \#8500 Quick Mount, Louisville, KY, USA) record was made to mount the maxillary cast on a semi-adjustable articulator (Whip Mix \#8645 Quick Mount, Louisville, KY. USA). The centric occluding relation was recorded by wax rims related by means of a silicone bite registration material (Genie Bite, Sultan Healthcare, Hackensack, NJ, USA) to mount the mandibular cast. This was followed by setting up of anatomic artificial teeth (VertexTM Quint teeth, Vertex- Dental, Netherland) then teeth try-in. After carrying out any necessary adjustments, processing of the trial dentures was then done as usual. Laboratory remounting was performed for all processed dentures by remounting the deflasked dentures in the laboratory using the split cast technique. Occlusion was corrected for processing changes to recapture the original vertical dimension of occlusion. ${ }^{6,35}$

For the clinical remounting (experimental) group, a plaster remount index was done for the processed maxillary dentures to preserve the orientation of the maxillary cast to the articulator and spare taking a new face bow record from the patient. ${ }^{35}$ 
All processed dentures were then finished and polished as usual. The two sets of dentures were inserted and delivered in the same appointment for each patient. At the day of denture insertion, the following steps were carried out similarly for all dentures. Denture borders and fitting surfaces were inspected for any sharp edges or nodules and adjusted accordingly. Denture adaptation was assessed using pressure indicating paste and any necessary adjustments were carried out to eliminate all pressure areas from the denture's fitting surface. Rugae areas and median palatine raphe were relieved when necessary. Retention and stability of the dentures were assessed intra-orally using the modified Kapur criteria (Olshan modification). ${ }^{36}$

\section{Occlusal assessment and adjustment procedures}

For group I dentures (intra-oral occlusal assessment using articulating paper method), 60 micron horseshoe-shaped articulating paper strips (Bausch KG, Köln, Germany) were used. ${ }^{37}$ Patients were asked to sit upright in the dental chair. With the dentures in place, an articulating paper strip was inserted intraorally so as to cover the occlusal surfaces of all mandibular denture teeth and held firmly. Patients were then asked to tap firmly through the articulation paper at least 5 times in succession. ${ }^{32}$ Patients were instructed to attempt to generate their perceived maximum occlusal force while tapping through the articulating paper strip. The maxillary and mandibular dentures were then removed from the patient's mouth and inspected for any premature and/or heavy contacts, which were adjusted by selectively grinding them using an acrylic bur and a straight hand piece. The procedure was repeated until even, simultaneous and sufficient number of contact marks was seen on the posterior teeth bilaterally. ${ }^{38}$ After each adjustment, the remaining marks were totally erased with alcohol swaps and the strip was replaced once perforated.

For group II dentures (clinical remounting method), the dentures were removed from the patient's mouth and dried carefully. Undercuts on the tissue sides of the maxillary denture were blocked out with silicon putty. ${ }^{5}$ The blocked out fitting surface was poured using hard stone to make the maxillary remount cast. The maxillary denture with its cast was then seated accurately in the previously constructed plaster remount index so that all denture teeth accurately and completely fit in their respective plaster indentations. Plaster was then used to mount the maxillary cast to the articulator. After complete setting of the plaster, the maxillary denture was removed from its cast, silicone was removed from the denture, and then the denture was replaced inside the patient's mouth to record the horizontal maxillomandibular relationship with the mandible in centric relation. To do so, the mandibular denture was dried thoroughly; wax (Aluwax Dental Products Co, Allendale, Mich) was immersed in a water bath at $52^{\circ} \mathrm{C}$ for 20 seconds and applied as a layer on the dried occlusal surface of the mandibular denture. The patient was instructed to guide the mandible into centric relation until contact is reached. The mandibular denture with the wax were then removed from the patient's mouth and chilled in water. The record was deemed unacceptable if the teeth had penetrated the wax. Once accepted, the procedure was repeated in the mouth by guidance into centric relation to check the record. The whole procedure was repeated if there was evidence of tipping of the maxillary denture upon closure or if the impressions (indentations) of different depths were recorded as this indicates uneven pressure. The record was approved when the maxillary denture teeth fit perfectly into the respective impressions of the wax on the mandibular denture. The dentures, attached to the final approved centric record, were then carefully removed from the patient's mouth. The undercuts of the mandibular denture base and tongue area were filled with putty silicone, then poured with hard stone to make the mandibular remount cast. The maxillary denture was replaced on its mounted cast. The mandibular 
denture (on its cast) was related to the maxillary denture by means of the centric record then mounted on the lower member of the articulator using plaster. After complete setting of the mounting plaster, the centric relation record was removed and 60 micron horseshoe-shaped articulating paper strips were used to detect the presence of premature and/or heavy contacts.

The following selective grinding rules ${ }^{35}$ were executed: a) The cusp tip was reduced when it contacted prematurely in centric and eccentric positions, otherwise the opposing fossa was deepened; b) The BULL rule (Buccal cusp of maxillary teeth and lingual cusp of mandibular teeth) was applied to working side prematurities, c) Correction of balancing side prematurities was done based on the judgement of which supporting cusp best maintained centric and then its antagonist was reduced. d) Protrusive balance was also checked by placing the articulating paper between the teeth and moving the articulator into the protrusive position: For anterior interferences, the labio-incisal surfaces of the mandibular anterior teeth and the palatal surfaces of the maxillary anterior teeth were grinded. Heavy contacts on the posterior teeth in the protrusive position were removed by grinding the distal inclines of the maxillary cusps and the mesial inclines of the mandibular cusps. Grinding procedures continued until there were smooth transitions as the articulator was moved from one occlusal position to another. ${ }^{6}$

\section{Computerized occlusal analysis}

After occlusal adjustment, computerized occlusal analysis for the two sets of dentures for each patient was carried out using the T-Scan III (Tekscan Inc., South Boston, MA, USA) system (Version 8). The system includes an electronically-charged, mylarencased, 100 microns thick recording sensor (Highdefinition Generation IV sensor, Tekscan Inc. S. Boston, MA, USA) (Fig. 1) that is scanned in 0.003 second time increments. Small and large-sized sensors are available. Sensor size was chosen to suit the patient's dental arch. Following manufacturer's recommendation, a proper sensitivity range was established before any occlusal data acquisition. ${ }^{30}$, ${ }^{39}$ Sensor conditioning procedures through 2 to 4 test closures ${ }^{21,23}$ were also done for each participant.

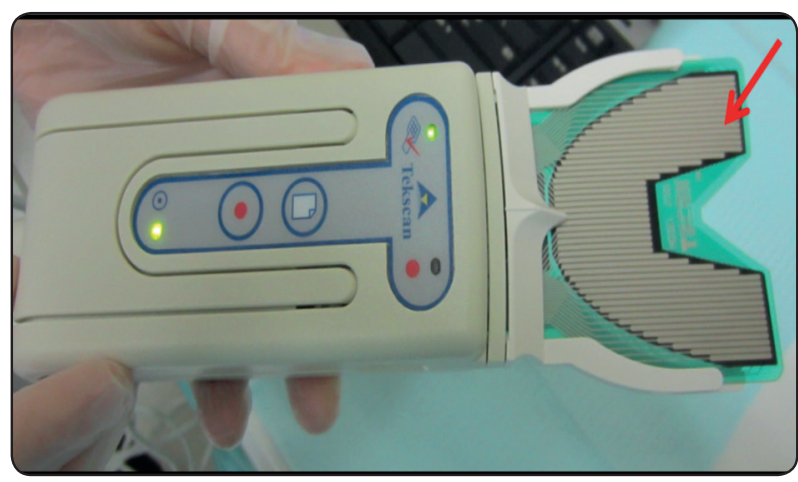

Fig. (1) T-Scan III sensor (Red Arrow) mounted in the T-Scan III holder and handle

For all T-Scan procedures, participants were asked to sit in a relaxed upright position in the dental chair. The sensor was consistently aligned so as to be parallel to the occlusal plane and centered on the midline between the central incisors. ${ }^{23}$

Occlusal parameters (OT and DT) were recorded for each set of dentures. To record the OT, each participant was instructed to firmly occlude into the sensor with their dentures until maximum intercuspation, hold their teeth together for 1 to 3 seconds, then disocclude and re-intercuspate into the sensor once more. The participant was asked to repeat this 3 to 4 times. To record DT, each participant was instructed to occlude on the sensor in centric, with normal pressure, till maximum intercuspation, hold their teeth together for 1 to 3 seconds then start a left or right excursion from that maximally intercuspated position then disocclude. ${ }^{23}$ The participant was asked to repeat this 3 to 4 times for the left excursion (DT-Left) and 3 to 4 times for the right excursion (DT-Right). The mean OT, DT-Right and DT-Left were then calculated by taking the average of the recordings. 
An occlusal analysis display for a group I denture (control group- articulating paper) and a group II denture (experimental group- clinical remounting) is shown in Figure $\mathbf{2}$ and Figure $\mathbf{3}$ respectively.

Statistical analysis was performed with SPSS 16.0 for Windows (SPSS Inc., Chicago, Illinois) software. Data were collected, tabulated, and presented as mean and standard deviation. Paired t-test was used to analyze the OT, DT-Right and DTLeft values. A probability value of less than 0.05 was considered statistically significant.

\section{RESULTS}

The stability and retention of all dentures ranged from good to very good so none of the dentures was left out and the results of all denture sets were included in the statistical analysis. Table $\mathbf{1}$ shows the mean occlusion (OT) and disocclusion (DT) times in both denture groups (methods). The mean recorded OT was 0.48 seconds in the intraoral method group and decreased to 0.32 seconds in the clinical remounting group. This decrease was statistically significant. The mean recorded DT was 0.6 and 0.62 seconds for the right and left excursions respectively in the intra-oral method group. However they decreased to 0.36 and 0.37 seconds in the clinical remounting group. Again this decrease was statistically significant for both right and left lateral excursions.

Table 1: Mean occlusion (OT), right and left disocclusion (DT) times for both methods

\begin{tabular}{|c|c|c|c|}
\hline & $\begin{array}{c}\text { Intra-oral method using articulating } \\
\text { paper (Group I dentures) } \\
n=20\end{array}$ & $\begin{array}{l}\text { Clinical Remounting Method } \\
\text { (Group II dentures) } \\
\mathbf{n = 2 0}\end{array}$ & \\
\hline Occlusion Parameter & Mean (SD) & Mean (SD) & P-value \\
\hline OT & $0.48(0.18)$ & $0.32(0.19)$ & $<0.001^{*}$ \\
\hline DT- Right & $0.60(0.17)$ & $0.36(0.14)$ & $<0.001^{*}$ \\
\hline DT- Left & $0.62(0.23)$ & $0.37(0.15)$ & $<0.001^{*}$ \\
\hline
\end{tabular}

*Significant at $p<0.05 ; n=$ number of dentures in each group

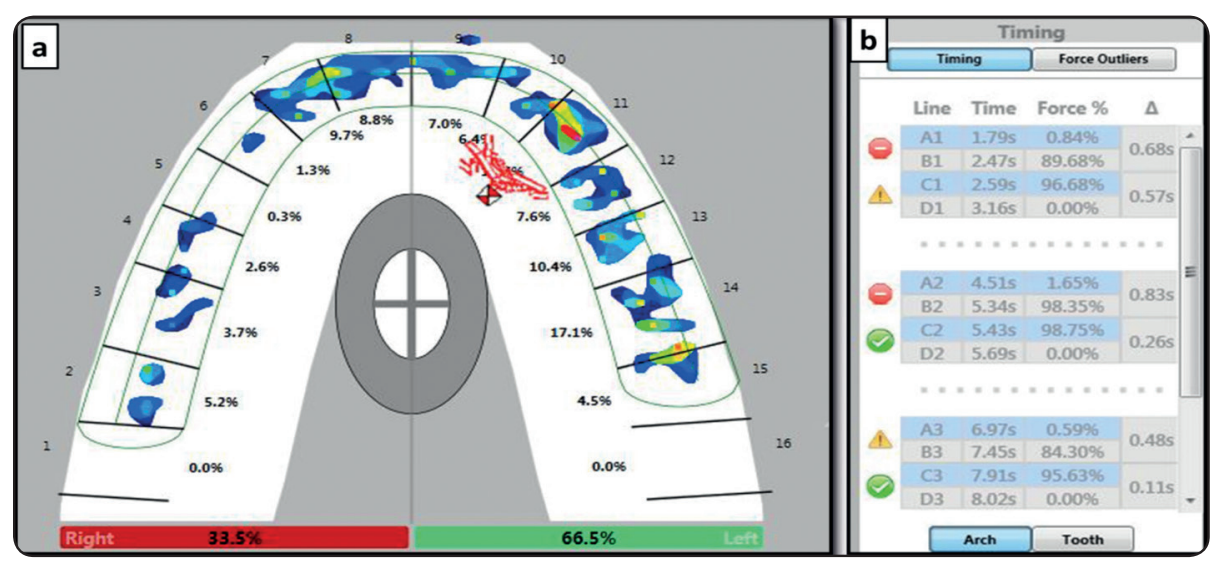

Fig. (2) Occlusal analysis of one of the dentures in Group I (Articulating paper method). (a) A 2-dimensional coloured view of the arch showing the centre of force marker (red \& white diamond) located outside the grey eclipse with unequal relative occlusal force distribution between the right (33.5\%) and left (66.5\%) sides. (b) Display of the values of the occlusion (AB) and disocclusion (C-D) times. Green mark denotes that it is within the acceptable clinical values, yellow mark denotes that it is border line and red mark denotes that it exceeded the acceptable clinical values. 


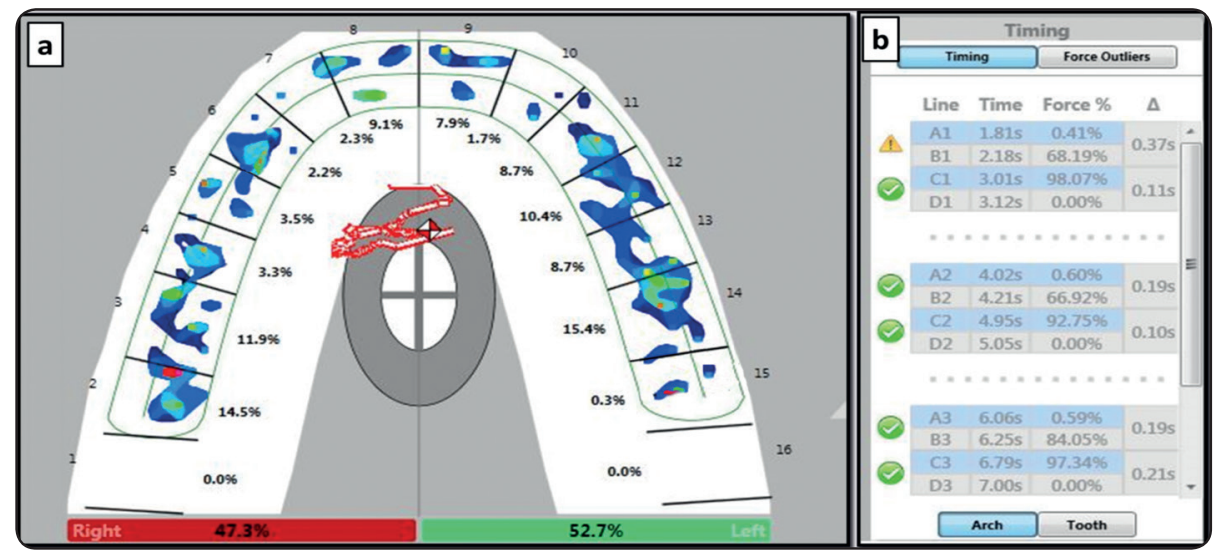

Fig. (3) Occlusal analysis of one of the dentures in Group II (Clinical Remounting Group). (a) A 2-dimensional coloured view of the arch showing the centre of force marker (red \& white diamond) located inside the grey eclipse with nearly equal relative occlusal force distribution between the right (47.3\%) and left (52.75\%) sides. (b) Display of the values of the occlusion (A-B) and disocclusion (C-D) times.

\section{DISCUSSION}

The results of the current study clearly reject the null hypothesis as there were highly significant differences between the two methods (denture groups) with the clinical remounting method showing significantly shorter mean occlusion and disocclusion times that approached or even reached the clinically acceptable values.

Before discussing the results in detail, it is worth mentioning that all laboratory work was carried out in the same lab by the same dental technician. This was necessary to exclude as much as possible technical and material related variables that could have affected the results. Similarly constructing two sets of dentures for the same patient in the same intraoral conditions was done in an attempt to exclude the influence of patient-related variables, such as the condition of the denture foundation area, chewing side preference and patient bite quality. ${ }^{40}$ Laboratory remounting was carried out for all dentures in both denture groups for two main reasons. First, to achieve a common baseline for all dentures by detecting and eliminating "processing" errors in all of them. Second, to ensure that any between-group differences detected will be related only and solely to the effect of clinical remounting as opposed to the more popular intraoral assessment method. The retention and stability of all dentures was assessed prior to any occlusal assessment and adjustment procedures. It is well established now that occlusion is directly correlated to denture stability. So it was essential to ensure that all dentures were initially retentive and stable enough so as not to have any negative influence on the results. This was indeed the case in the current research as the retention and stability of all dentures ranged from good to very good. All previously mentioned factors helped in attaining more reliable and valid results based on which solid conclusions could be drawn.

The results of the study revealed that the mean OT was 0.48 seconds and 0.32 seconds for group I and II dentures respectively. The mean DT was 0.60 seconds and 0.62 seconds for group I dentures while it decreased significantly to 0.36 seconds and 0.37 seconds in group II dentures. Direct comparison of these values with those in other studies was not possible because to the best of the author's knowledge there are no similar researches in the dental literature that compared OT and DT times between clinically remounted versus non- 
clinically remounted dentures. However, there was a clinical study ${ }^{27}$, that used the T-scan II occlusal analysis system to compare between old and newly constructed conventional complete dentures. The authors reported an average of $0.42 \mathrm{~s}, 0.48 \mathrm{~s}$ and $0.45 \mathrm{~s}$ for OT, DT-right and DT-left times respectively for newly constructed dentures and $0.43 \mathrm{~s}, 0.56 \mathrm{~s}$ and $0.51 \mathrm{~s}$ OT, DT-right and DT-left times respectively for the old dentures worn by the same patients. Direct comparison between these values and the current study values is not possible due to the difference in the T-Scan version used in both studies and because it was not clearly stated whether their dentures were clinically remounted or not. However the values recorded in the current study are still somehow close to the value range reported in the aforementioned study. This implies that the T-scan recordings of the current study do not outlie those reported for conventional complete dentures in the dental literature and hence could be considered as valid and reliable.

As mentioned earlier, shorter occlusion times indicates the presence of fewer pre-maturities as less time is required for all teeth to contact as the patient closes into maximum intercuspation, hence demonstrating more occlusal contact simultaneity and equilibration. The results revealed that clinical remounting procedures significantly shortened occlusion and disocclusion times indicating that a more harmonious and balanced occlusion was achieved. This means that the occlusal adjustment that was carried out based on that method was much more accurate and precise than that based on the intra-oral articulating paper method. This objective finding was furtherly evident from the force distribution pattern and centre of force (COF) trajectory displayed in the 2-dimensional T-Scan views as seen in figures 2 and 3 . The COF marker pinpoints the location of the sum of the total force of the occlusal contacts. This is done by calculating the sum of the medio-lateral and antero-posterior force moments of the recorded contacts. The COF marker is related to a dual elliptical target which represents the ideal location of the COF for any maximum intercuspation closure, and acts as a guide with respect to a normal occlusion. The outer eclipse (grey zone) shows the area within which the COF of $95 \%$ of a normal population is found. While the inner (white) zone shows the area within which the COF of $68 \%$ of a normal population is found. ${ }^{41}$ As seen from the figures that represented a denture from each group (method), clinical remounting resulted in a "nearly" equal force distribution pattern between the right (47.3\%) and left (52.75\%) sides of the arch that approached a 50-50\% situation (Fig. 3). The COF marker also lied in the outer (grey) eclipse zone. This means that the occlusion achieved with clinically remounted dentures is within the $95 \%$ range of normal occlusion. On the other hand occlusal adjustment based on the intra-oral articulating method led to a less balanced occlusion with an unequal force distribution pattern between the two sides of the arch $(33.5 \%$ (left) and $66.5 \%$ (right)) (Fig. 2). The COF marker was located outside the grey eclipse towards the side with the heaviest occlusal contacts, again indicating uneven occlusal balance.

Previous studies did report that although intraoral occlusal adjustment based on articulating paper marks may be indeed faster and is more common yet it is much less accurate than occlusal correction based on registering a centric relation record and remounting the finished dentures. ${ }^{3,5,12,16,17}$ However all these studies depended on subjective findings and personal interpretations. The results of the current study now "objectively" prove and confirm their subjective statements. The outcomes of the current study also explain the results of Jandial et al ${ }^{10}$ who found a highly significant relationship between the absence of clinical remounting and occlusal disharmony. The results of the current study could be attributed to several factors. One possible factor is that in the intra-oral assessment 
method, the salivary impregnation of the denture surfaces, which can diffuse the articulating paper ink, could have led to false positives ${ }^{40}$ Other factors including eccentric closures by the patient, tissue distortions and shifting of the denture bases on the resilient tissues may have prevented the articulator paper marks from accurately recording occlusal errors; ${ }^{3,5,38}$ in fact it has been reported that it may actually increase the amount of occlusal errors. ${ }^{3} \mathrm{On}$ the contrary, in the clinical remounting procedures, properly made interocclusal records will not cause the denture bases to slip or rotate in relation to their bony foundations. When these records are then used to accurately remount the dentures on the articulator, the dentures will be firm on their remount casts allowing for more "precise detection" and hence subsequent "correct adjustment" of occlusal errors. ${ }^{3,14}$ Not to mention also the great advantage of working in the absence of saliva which certainly allows for better visibility of the markings and therefore more refined adjustment. ${ }^{16}$

A more in-depth analysis of the results reveal that although clinical remounting significantly shortened the occlusion time, "both" methods still did not reach the clinical goal of 0.2 seconds. While searching the literature, the author found a clinical study ${ }^{28}$ that used the T-Scan III system and reported an average of $0.69 \mathrm{~s}$ for OT in normal dentate subjects with healthy TMJs. Their values are higher than those reported in the present study and their results cannot be directly compared to the present results due to differences in the dentition state. However, it could be used to support the assumption that the values obtained in this study, although do not reach the recommended values, still did not exceed values obtained for natural dentitions with healthy TMJs. This infers that OT values and consequently occlusal balance achieved by both methods could still be considered within the clinically tolerable levels. So it is accordingly fair to say that the intra-oral assessment method could still be used to achieve "clinically-tolerable" occlusal balance. However based on the results it is recommended to perform clinical remounting whenever feasible and possible.

It is noteworthy that in this research, clinical remounting was performed on the day of insertion as recommended by several researchers. 7, 12, 38 However, could the OT values decrease even more if clinical remounting was repeated again 8 to 10 days post-insertion after denture settling? This remains an un-answered question that could be the trigger for future research.

As for the disocclusion time, it was found that a DT that is less than 0.5 was accompanied by a significant decrease in contractile muscle activity to near resting state values during mandibular excursions. This in turn reduces the stress on the temporomandibular joints and consecutively maintains their health. ${ }^{23,31,39}$ In the current study, clinical remounting significantly decreased the DT to 0.36 seconds (DT-Right) and 0.37 seconds (DTLeft). These values were not only less than the 0.4 seconds that represent the ideal clinical goal but were also way less than the $0.79 \mathrm{~s}$ and $0.91 \mathrm{~s}$ that were recorded for DT-right and DT-left respectively in normal dentate subjects with healthy TMJs. ${ }^{28}$ These are very promising results and emphasize the importance of clinical remounting procedures in complete denture construction.

\section{CONCLUSION}

Within the limitations of this study, it could be concluded that occlusal adjustment based on clinical remounting procedures is more accurate and achieves more occlusal balance, as represented by shorter occlusion and disocclusion times, when compared to the intra-oral adjustment method using an articulating paper. 


\section{REFERENCES}

1. Oh WS, Saglik B. Remount cast fabrication for occlusal equilibration against artificial denture teeth. J Prosthet Dent. 2009;101(5):350-351.

2. Firtell DN, Finzen FC, Holmes JB. The effect of clinical remount procedures on the comfort and success of complete dentures. J Prosthet Dent 1987 ; 57(1):53-57.

3. Zarb GA, Bolender CL, Eckert SE, Jacob RF, Fenton AH, Merickse-Stern R. Prosthodontic treatment for edentulous patients: complete dentures and implant-supported prostheses. 12th ed. St. Louis: Mosby; 2003 . p. 399-414.

4. Liu FC, Luk KC, Suen PC, Tsai TS, Ku YC. Modified split-cast technique: a new, timesaving clinical remount technique. J Prosthodont. 2010;19(6):502-506

5. Badel T, Pandurić J, Kraljević S, Dulcić N. Checking the occlusal relationships of complete dentures via a remount procedure. Int J Periodontics Restorative Dent. 2007;27(2):181-192.

6. Shigli K, Angadi GS, Hegde P. The effect of remount procedures on patient comfort for complete denture treatment. J Prosthet Dent 2008; 99: 66-72.

7. Al-Quran FA. A clinical evaluation of the clinical remount procedure. J Contemp Dent Pract. 2005;6(1):48-55.

8. Azarian M, Khoubivand M, Ghaffari S, Eghtedari N. Improving satisfaction of complete denture through clinical remount correction of occlusion. Bull. Env. Pharmacol, Lif Sci. 2015; 4 (2): 151-155.

9. Polyzois GL, Karkazis HC, Zissis AJ. Remounting procedures for complete dentures: a study of occlusal contacts by the photocclusion technique. Quintessence Int 1991;22:811-815.

10. Jandial S, Kotwal B, Gupta R, Mahajan N, Kharyal S, Kotwal V. The Incidence of Occlusal Disturbances and its Causes in Complete Denture Patients. International Journal of Scientific Study. 2017 ; 5 (6): 217-220.

11. Harrison A, Huggett R, Murphy WM. Complete denture construction in general dental practice: an update of the 1970 survey. Br Dent J 1990; 169: 159- 163.

12. Hochstedler JL, Shannon JL. A time-saving method for performing a clinical remount procedure with complete dentures. J Prosthet Dent. 1995 ;74(1):39-41.

13. Petropoulos VC, Rashedi B: Complete denture education in U.S. dental schools. J Prosthodont 2005;14:191-197.
14. Ansari IH. Simplified clinical remount for complete dentures. J Prosthet Dent 1996;76:321-324.

15. Okuma K, Hirano S, Hayakawa I. Occlusal pressure pattern analysis of complete dentures for evaluation of occlusal adjustment. J Med Dent Sci 2004; 51:197-203.

16. Lytle RB. Complete denture construction based on a study of the deformation of the underlying soft tissues. J Prosthet Dent 1959;9:539-551.

17. Wilson J, Rees JS. Comparison of interocclusal contacts registered intraorally and after a remount procedure in complete denture patients. Eur J Prosthodont Restor Dent. 2006;14(4):146-150.

18. Mpungose SK, Geerts GA. Analyzing Complete Denture Occlusal Contacts: Accuracy and Reliability. Int J Prosthodont. 2016;29(1):50-52.

19. Maness WL, Benjamin M, Podoloff R, Bobick A, Golden RF. Computerized occlusal analysis: a new technology. Quintessence Int 1987; 18:287-292.

20. Koos B, Godt A, Schille C, Göz G. Precision of an instrumentation-based method of analysing occlusion and its resulting distribution of forces in the dental arch. J Orofac Orthop 2010; 71:403-410.

21. Martins MJS, Caramelo FJ, Ramalho da Fonseca JA, Nicolau PMG. In vitro study on the sensibility and reproducibility of the new T-Scan III HD system. Rev Port Estomatol Med Dent Cir Maxilofac 2014; 55: 14-22.

22. Kerstein RB, Thumati P, Padmaja S. Force finishing and centering to balance a removable complete denture prosthesis using the T- Scan III computerized occlusal analysis system. J Indian Prosthodont Soc 2013; 13:184188

23. Kerstein RB, Radke J. The effect of disclusion time reduction on maximal clench muscle activity levels. Cranio 2006; 24: 156-165.

24. Carey JP, Craig M, Kerstein RB, Radke J. Determining a relationship between applied occlusal load and articulating paper mark area. Open Dent J 2007; 1:1-7

25. Cohen-Levy J, Cohen N. Computerized analysis of occlusal contacts after lingual orthodontic treatment in adults. Int Orthod 2011; 9:410-431.

26. Kerstein RB, Radke J. Clinician accuracy when subjectively interpreting articulating paper markings. Cranio. 2014;32(1):13-23. 
27. Sierpinska T Golebiewska M, Kuc J, Lapuc M. The influence of the occlusal vertical dimension on masticatory activities and hyoid bone position in complete denture wearers. Adv Med Sci 2009; 54:104-108.

28. Haralur SB. Digital evaluation of functional occlusion parameters and their association with temporomandibular disorders. J Clin Diagn Res 2013; 7: 1772-1775.

29. Gümüş HÖ, Kılınç Hİ, Tuna SH, Ozcan N. Computerized analysis of occlusal contacts in bruxism patients treated with occlusal splint therapy. J Adv Prosthodont 2013; 5:256-261

30. Kerstein RB, Grundset K. Obtaining Bilateral Simultaneous Occlusal Contacts with Computer Analyzed and Guided Occlusal Adjustments. Quintessence Int 2001; 32:7-18

31. Kerstein RB, Wright N. An Electromyographic and Computer Analysis of Patients Suffering from Chronic Myofascial Pain Dysfunction Syndrome; Pre and Post Treatment with Immediate Complete Anterior Guidance Development. J Prosthet Dent 1991; 66:677 - 686.

32. Qadeer S, Kerstein R, Kim RJ, Huh JB, Shin SW. Relationship between articulation paper mark size and percentage of force measured with computerized occlusal analysis. J Adv Prosthodont. 2012;4(1):7-12

33. Boening KW, Walter MH. Computer-aided evaluation of occlusal load in complete dentures. J Prosthet Dent. 1992; 67(3):339-344.
34. McGarry TJ, Nimmo A, Skiba JF, Ahlstrom RH, Smith $\mathrm{CR}$, Koumjian JH: Classification system for complete edentulism. J Prosthodont 1999; 8: 27- 39.

35. Hassaballa MA: Clinical Complete Denture Prosthodontics. King Saud University, Saudi Arabia, 2004 pg. 345-358

36. Olshan AM, Ross NM, Mankodi S, Melita S. A modified Kapur scale for evaluating denture retention and stability: methodology study. Am J Dent 1992; 5:88-90.

37. Atashrazm P, Ansari Lari H, Khorsand M. An Evaluation of Occlusal Contacts of Remounted Complete Denture before Final Occlusal Adjustment. Shiraz Univ Dent J 2009; 9: 1-5.

38. Gutowsk A. Remounting and occlusal adjustment of complete dentures. J Gnathol. 1990; 9: 9-22.

39. Kerstein RB. Disocclusion time-reduction therapy with immediate complete anterior guidance development to treat chronic myofacial pain-dysfunction syndrome. Quintessence Int 1992; 23:735-747.

40. Kerstein RB. Articulating paper mark misconceptions and computerized occlusal analysis technology. Dent Implantol Update 2008; 19:41-46

41. Teckscan Inc. T-scan III user manual: computerized occlusal analysis system. 2012 